\title{
Ventilator associated pneumonia in the ICU: microbiological profile
}

\begin{abstract}
Ventilator-associated pneumonia (VAP) refers to pneumonia which occurs in people who required mechanical ventilation through an endotracheal or tracheostomy tube for at least 48 hours. The study includes patients of pneumonia who were on mechanical ventilation for more than 48hours and admitted to the intensive care unit (ICU). During the study period 48 patients developed VAP out of which 51 isolates were recovered of which $66.7 \%$ were male and $33.3 \%$ were female. The incidence of early onset VAP was $19 \%$ while late onset VAP was $81 \%$. Acinetobacter species followed by Pseudomonas aeruginosa were the most common organisms causing early onset VAP. However, Acinetobacter spp. followed by Klebsiella spp. were the common organisms causing late onset VAP. Acinetobacter spp. was overall the most common isolate (66\%) having $100 \%$ resistance to ampicillin, amikacin, ciprofloxacin, cefotaxime and cefepime. Pseudomonas spp. showed $100 \%$ resistance to gentamicin, ceftazidime and piperacillin. ESBL (Extended spectrum $\beta$ lactamase) production was detected in $22 \%$ of the isolates with Enterobacter spp being the most common producer $(50 \%) .80 \%$ of the isolates were carbapenemase producers, $74 \%$ showed MBL (Metallo $\beta$ lactamase) production and $40 \%$ were AmpC producers. VAP due to multidrug resistant organisms (MDRO) is one of the most dreadful complications that can occur in the critical care setting. Various strategies such as strict infection control measures, judicious prescribing of antibiotics, antibiotic resistance surveillance programs and antibiotic cycling are crucial in controlling infections due to these bacteria in patients admitted to ICU.
\end{abstract}

Keywords: VAP, ICU, ESBL, MBL, AmpC
Volume 4 Issue 5 - 2017 Geetika Rana, Shweta Sharma, Charoo Hans
Department of Microbiology, RML Hospital \& PGIMER, India

Correspondence: Shweta Sharma, Senior Resident, Department of Microbiology, RML Hospital \& PGIMER, New Delhi, India, Email drshwetamicro@gmail.com

Received: January 28, 2017| Published: May II, 2017
Abbreviations: VAP, ventilator-associated pneumonia; ICU, intensive care unit; HAP, hospital acquired pneumonia; CPIS, chronic pulmonary infection score; MIC, minimum inhibitory concentration; ESBL, extended spectrum beta lactamase production; DDST, double disc synergy testing; MBL, metallo beta lactamase

\section{Introduction}

Ventilator associated pneumonia (VAP) is defined as nosocomial pneumonia in patients on mechanical ventilator support (by endotracheal tube or tracheostomy) for $>48 \mathrm{~h}$ during their ICU stay, excluding any infection present or in incubation at the time of ICU admission. ${ }^{1}$ VAP is estimated to occur in $9-27 \%$ of all mechanically ventilated patients, with the highest risk being early in the course of hospitalization. ${ }^{2,3}$ It is the second most common nosocomial infection in the intensive care unit (ICU) and the most common in mechanically ventilated patients. ${ }^{4,5}$ The mortality with VAP is considerably high, varying from 24 to $50 \%$ and can reach as high as $76 \%$ in some specific settings. ${ }^{6}$

The etiologic agents widely differ according to the population of patients in an intensive care unit, duration of hospital stay, prior antimicrobial therapy and co-morbid conditions. Despite the advancements in antimicrobial regimes, VAP continues to be an important cause of morbidity and mortality. Inadequate antimicrobial therapy, such as inappropriate antimicrobial coverage, or delayed initiation of antimicrobials has been associated with higher hospital mortality in subjects with hospital acquired pneumonia (HAP) or VAP. ${ }^{7}$

Therefore the aim of this study was to analyse the microbiological and clinical profile of VAP in our hospital, risk factors and prevalence of multi-drug resistant organisms so as to implement effective prevention strategies.

\section{Material and methods}

The study was conducted during the period of Jan 2014 to Dec 2014 in the ICUs of a large tertiary care hospital in North India. All mechanically ventilated patients developing pneumonia after $>48$ hrs of ventilation were included in the study. To diagnose VAP in the patients, Chronic Pulmonary Infection Score (CPIS) was used. ${ }^{8}$ Patients were screened for 1) New or persistent pulmonary infiltrates appeared on chest radiograph not otherwise explained. 2) fever 3) Leucocytosis 4) Oxygenation $\mathrm{PaO}_{2} / \mathrm{Fio} 2$ 5) Purulent respiratory secretions. Early onset VAP was defined as pneumonia that occurred within 4days of intubation whereas late onset VAP defined as pneumonia after 4days of intubation. Only patients exhibiting bacteriologically documented pneumonia were studied; establishment of etiologic diagnosis required isolation of bacteria in significant quantity from samples of lower respiratory tract secretions (endotracheal secretions $>105 \mathrm{cfu} /$ $\mathrm{ml}$, protected brush catheter $>103 \mathrm{cfu} / \mathrm{ml}$ and bronchoalveolar lavages $>104 \mathrm{cfu} / \mathrm{ml}$ ) or isolation of a definitive pathogen from a blood or pleural fluid culture. ${ }^{9}$

The microbiological samples were collected and processed according to standard protocols. ${ }^{10}$ All the bacteria isolated were identified to the species level by standard biochemical tests and their antibiotic susceptibility testing was performed by the Kirby-Bauer disc diffusion method on Muller-Hinton agar as per the Clinical and Laboratory Standards Institute guidelines. Minimum inhibitory concentration (MIC) was determined by E-test. 
Extended Spectrum Beta Lactamase production (ESBL) was determined by double disc synergy testing (DDST) using antibiotic disc of ceftazidime $30 \mu \mathrm{g}$ and ceftazidime-clavulanic acid 30/10 $\mu \mathrm{g}$, also cefotaxime $30 \mu \mathrm{g}$ and cefotaxime-clavulanic acid 30/10 $\mu \mathrm{g} .{ }^{11}$ Strains resistant to carbapenems were tested for carbapenemase production by Modified Hodge test. Metallo beta lactamase (MBL) production was determined by using Disc Potentiation test by using Imipenem disc $10 \mu \mathrm{g}$ and Imipenem-EDTA disc. ${ }^{12}$ Isolates were screened for AmpC $\beta$ - lactamases by standard disc diffusion breakpoint for cefoxitin. Isolates with zone diameter less than $18 \mathrm{~mm}$ for cefoxitin were tested for AmpC activity by Disc potentiation test by using Cefotaxime $30 \mu \mathrm{g}$ disc and cefotaxime- 3 amino phenylboronic acid $30 \mu \mathrm{g} / 300 \mu \mathrm{g}$ disc. ${ }^{13,14}$

Table I Antimicrobial resistance profile of isolates from VAP

\section{Results}

During the study period, 48 patients developed VAP out of which 51 isolates were recovered. $66.7 \%$ were male and $33.3 \%$ were female. $55 \%$ patients had undergone a surgery while $45 \%$ were admitted for medical reasons. The incidence of early onset VAP was $19 \%$ while late onset VAP was $81 \%$. Acinetobacter species followed by Pseudomonas aeruginosa were the most common organisms causing early onset VAP. While Acinetobacter spp. followed by Klebsiella spp. were the most common organisms causing late onset VAP. Acinetobacter spp. was overall the most common isolate (66\%) having $100 \%$ resistance to ampicillin, amikacin, ciprofloxacin, cefotaxime and cefepime. Only $8.8 \%$ were sensitive to imipenem with MIC value ranging from 0.25 to $1.5 \mathrm{mcg} / \mathrm{ml}$ and breakpoint MIC (Microscan) $<4 \mathrm{mcg} / \mathrm{ml}$ (Table 1).

\begin{tabular}{|c|c|c|c|c|c|c|}
\hline \multicolumn{2}{|c|}{ Acinetobacter ${ }^{34}$} & \multirow{2}{*}{$\begin{array}{l}\text { Pseudomonas }^{6} \\
:\end{array}$} & \multirow{2}{*}{$\begin{array}{l}K^{K l e b s i e l l a}{ }^{5} \\
5(100 \%)\end{array}$} & \multirow{2}{*}{$\begin{array}{l}\text { Enterobacter }{ }^{4} \\
4(100 \%)\end{array}$} & \multirow{2}{*}{$\begin{array}{l}\text { E. coli' } \\
\text { I(I00\%) }\end{array}$} & \multirow[t]{2}{*}{ S. aureus' } \\
\hline A & $34(100 \%)$ & & & & & \\
\hline G & $33(97 \%)$ & $6(100 \%)$ & $5(100 \%)$ & $3(75 \%)$ & $0 \%$ & \\
\hline Ak & $34(100 \%)$ & $6(100 \%)$ & $5(100 \%)$ & $3(75 \%)$ & & \\
\hline $\mathrm{Cf}$ & $34(100 \%)$ & $6(100 \%)$ & $5(100 \%)$ & $3(75 \%)$ & $\mathrm{I}(100 \%)$ & $\mathrm{I}(100 \%)$ \\
\hline $\mathrm{Ce}$ & $34(100 \%)$ & $:$ & $5(100 \%)$ & $3(75 \%)$ & $\mathrm{I}(100 \%)$ & \\
\hline $\mathrm{Ca}$ & $:$ & $5(83.3 \%)$ & $:$ & $:$ & $:$ & \\
\hline $\mathrm{Pc}$ & $:$ & $6(100 \%)$ & $:$ & $:$ & $:$ & \\
\hline PT & $32(100 \%)$ & $5(83.3 \%)$ & $5(100 \%)$ & $3(75 \%)$ & $\mathrm{I}(\mathrm{I00 \%})$ & \\
\hline \multicolumn{7}{|c|}{ Cfp } \\
\hline $\operatorname{Imp}$ & $3 \mid(9 \mid .2)$ & $3(50 \%)$ & $4(80 \%)$ & $3(75 \%)$ & $\mathrm{I}(100 \%)$ & \\
\hline Ox & $:$ & $:$ & $:$ & $:$ & $:$ & $\mathrm{I}(100 \%)$ \\
\hline $\mathrm{T}$ & & & & & & $0 \%$ \\
\hline$E$ & & & & & & $\mathrm{I}(100 \%)$ \\
\hline Co & & & & & & $\mathrm{I}(100 \%)$ \\
\hline $\mathrm{Va}$ & & & & & & $0 \%$ \\
\hline
\end{tabular}

Note: A, ampicillin; G, gentamicin; Ak, amikacin; Cf, ciprofloxacin; Ce, cefotaxime; Ca, ceftazidime; Pc, piperacillin; PT, piperacillin tazobactam; Cfp, cefoperazone; Imp, imipenem; Ox, oxacillin; T, tetracycline; E, erythromycin; Co, cotrimoxazole; Va, vancomycin

Pseudomonas spp. showed $100 \%$ resistance to gentamicin, ceftazidime and piperacillin. $16.7 \%$ were sensitive to piperacillintazobactam and $50 \%$ to imipenem (Table 1). The MIC for sensitive strains were found to be $12 \mathrm{mcg} / \mathrm{ml}$ for piperacillin-tazobactam and
$38-1.5 \mathrm{mcg} / \mathrm{ml}$ for imipenem. The breakpoint MIC were $<16 \mathrm{mcg} / \mathrm{ml}$ and $<4 \mathrm{mcg} / \mathrm{ml}$ respectively. The AMR pattern of all the causative isolates is given in Table 2. No discrepancies were found in results from MIC e test and disc diffusion test.

Table 2 Drug resistance among various isolates

\begin{tabular}{llllll}
\hline Isolate & No. (\%) & ESBL producer (\%) & Amp C producers (\%) & Carbapenamase producer by MHT (\%) & MBL producer (\%) \\
\hline Acinetobacter & $34(66.7)$ & $8(23.5)$ & $16(47.1)$ & $29(85.3 \%)$ & $28(82.4)$ \\
Pseudomonas & $6(11.8)$ & $0(0)$ & $0(0)$ & $3(50)$ & $2(33.3)$ \\
Klebsiella & $5(9.8)$ & I (20) & $3(60)$ & $4(80)$ & $4(80)$ \\
Enterobacter & $4(7.8)$ & $2(50)$ & I (25) & $3(75)$ & $2(50)$ \\
E.coli & I (2.0) & $0(0.0)$ & $0(0)$ & I (100) & I (100) \\
S. aureus & I (2.0) & $:$ & $:$ & $:$ & 4 \\
Total & $51(100)$ & II (22) & $20(40)$ & $40(80)$ & $37(74)$
\end{tabular}


ESBL production was detected in $22 \%$ of the isolates with Enterobacter spp being the most common producer (50\%). $80 \%$ of the isolates were carbapenemase producers. $100 \%$ of E. coli, $85 \%$ of Acinetobacter spp. and $80 \%$ of Klebsiella spp. showed carbapenemase production. $74 \%$ of the isolates showed MBL production. All of $E$. coli and $82 \%$ Acinetobacter spp. showed MBL production (Table 2). $40 \%$ of the isolates were AmpC producers. Klebsiella spp. (60\%) was the most dominant producer followed by Acinetobacter spp. (47\%) (Table 2).

\section{Discussion}

VAP is the most common nosocomial infection in the intensive care unit (ICU) with an incidence ranging from 8 to $28 \%$ in intubated mechanically ventilated patients. It is an important cause of morbidity and mortality despite the available antimicrobial therapy, advanced supportive care modalities, and the use of a wide-range of preventive measures. $^{1-5}$ Of the 48 patients diagnosed with VAP as per the CPIS score, $66.7 \%$ were male and $33.3 \%$ were female. The risk of pneumonia in patients receiving mechanical ventilation increases with the duration of ventilation. Fagon et al. ${ }^{15}$ showed that incidence of VAP rises with number days of mechanical ventilation. ${ }^{15}$ In our study $19 \%$ of the patients were categorized as early onset VAP while $81 \%$ as late onset VAP. In our ICU set up late onset VAP was most common when compared to early onset VAP, which correlated with the findings by Valles et al., ${ }^{16}$ where $27.5 \%$ of the patients had early-onset VAP and $72.5 \%$ had late-onset VAP. ${ }^{16}$ While in a study by Rello et al., ${ }^{17}$ the incidence of early onset VAP was $12.8 \%$ which is lower than our study.$^{17}$ Early-onset VAP is usually due to the under- lying pathology. On the other hand, late-onset VAP could be due to prolonged ventilation, evolution of the underlying disease, quality of nursing care, duration of antibiotic exposure or environmental ecology of the hospital. Studies have shown that previous antibiotic usage decreases early-onset VAP but markedly increases multidrug-resistant (MDR) pathogens.

In our study Acinetobacter species (66\%), Pseudomonas aeruginosa $(12 \%)$ and Klebsiella pneumoniae $(10 \%)$ were the most common organisms VAP, which is similar to Dey et al., ${ }^{18}$ where Acinetobacter species and Pseudomonas aeruginosa accounted to $48.94 \%$ and $25.53 \%$ respectively. ${ }^{18}$

$100 \%$ of Acinetobacter spp., Pseudomonas spp. and Klebsiella $s p p$. were multi-drug resistant. VAP due to MDR organisms is one of the most ominous complication leading to therapeutic failures, prolonged hospital stay, increased cost, morbidity and mortality.

ESBL belonging to groups SHV, TEM, CTX-M have mainly been implicated in the transfer of drug resistance in gram negative organisms. Initially these enzymes were commonly found in Klebsiella species and Escherichia coli, but now these enzymes have been described for most of the members of Enterobacteriaceae and few other gram negative non fermenting bacilli. ${ }^{19}$ In the present study, $22 \%$ of GNB were identified as ESBL producers. $50 \%(2 / 4)$ of Enterobacter spp. and 23.5\% (8/34) of Acinetobacter spp. were the major producers. Similar results were shown in a 2year study about changing In antimicrobial resistance pattern of isolates from an ICU by Sachin Jain et al. ${ }^{20}$

This study there was a high prevalence of AmpC beta lactamase among the study isolates $(40 \%)$. AmpC production among Enterobacteriaceae was highest in K. pneumoniae $(60 \%)$, while in that of nonfermenters, it was highest in Acinetobacter spp. (47.1\%). This may be due to the presence of plasmid mediated AmpC in the bacterias. In the present study $4.65 \%$ of gram negative bacteria $(20 \%$ Klebsiella spp and 4\% Acinetobacter spp.) were seen harbouring both AmpC beta lactamases and ESBL. In a study done by Dalela G et al., ${ }^{21}$ the prevalence of ESBL and AmpC $\beta$-lactamase and the coexistence of the phenotype (ESBL+AmpC $\beta$-lactamase) was found to be $66.9 \%$, $21.1 \%$ and $3.5 \%$ respectively. ${ }^{21}$ The coexistence of both enzyme types in the same strain not only results in elevated cephalosporin MICs but may also give false negative tests for the detection of ESBLs. The likely explanation is that AmpC-type beta-lactamases resist inhibition by clavulanate and hence obscure the synergistic effect of clavulanate and cephalosporins against ESBLs. Since, the detection of AmpC in our present study is so high, it may cause false negative detection rates for ESBL in other bacteria. ${ }^{22}$

Amongst the options that are available for the treatment of multi drug resistant organisms and ESBL producers, carbapenems constitute the drug of choice, but carbapenem resistance is rising alarmingly in isolates from intensive care units. The emergence of carbapenem-resistant micro-organisms severely limits the treatment options. In this study, $80 \%$ of the isolates were Carbapenemase producers and $74 \%$ were MBL producers. $85 \%$ of Acinetobacter spp. were carbapenemase producers and $82 \%$ were MBL producers. The clinical utility of Carbapenems is under threat with the emergence of acquired carbapenemases, particularly, metallobeta lactamases with the worldwide increase in occurrence, types and rate of dissemination of MBLs, early detection is critical. The detection of MBL and other carbapenemases is of utmost importance in deciding the most appropriate therapeutic regimen for the treatment. MBL producing GNB have now been reported in many geographical regions. Their ability to rapidly disseminate within an institution, leading to poor outcome, is a major concern. Enterobacteriaceae such as Klebsiella $s p p$., and E. coli, often carry hidden MBL genes. Such a scenario causes untoward clinical and infection control consequences. Hence, more sensitive and specific means of lab detection of MBL producing isolates is required to prevent the ongoing spread of the rapidly disseminating pathogens. ${ }^{23}$

The coexistence of different classes of $\beta$-lactamases in a single bacterial isolate may pose diagnostic and treatment challenges. The AmpC producing organisms can act as a hidden reservoir for the ESBLs. Also, the high-level expression of the AmpC $\beta$-lactamases may mask the recognition of the ESBLs and it may result in a fatal and an inappropriate antimicrobial therapy. The increase in the prevalence of the AmpC, MBL and the ESBL producing isolates may be indicative of the ominous trend of more and more isolates acquiring the resistance mechanisms, thus rendering the antimicrobial armamarium ineffective. ${ }^{24}$

The alarmingly high rates of multi-drug resistant organisms causing VAP in ICUs along with the ominous presence of ESBL, AmpC, carbapenamase and metallo-beta lactamase in them, suggest a situation demanding an intervention of infection control experts, hospital administration and policy planners to immediately introduce corrective and preventive actions to avoid a situation akin to post antibiotic era where even common infections will no longer have a cure and progress to unabated killings.

\section{Acknowledgements}

None.

\section{Conflict of interest}

The author declares no conflict of interest. 


\section{References}

1. Tablan OC, Anderson LJ, Besser R, et al. Guidelines for preventing health-care-associated pneumonia, 2003:recommendations of CDC and the Healthcare Infection Control Practices Advisory Committee. MMWR Re-comm Rep. 2004;53(RR-3):1-36.

2. Vincent JL, Bihari DJ, Suter PM, et al. The prevalence of nosocomial infection in intensive care units in Europe. Results of the European Prevalence of Infection in Intensive Care (EPIC) Study. EPIC International Advisory Committee. JAMA. 1995;274(8):639-644.

3. Chastre J, Fagon JY. State of the art: ventilator-associated pneumonia. Am J Respir Crit Care Med. 2002;165(7):867-903.

4. Hunter JD. Ventilator associated pneumonia. BMJ. 2012;344(e3325):e3325.

5. Afshari A, Pagani L, Harbarth S. Year in review 2011: Critical care infection. Crit Care. 2012;16:242-247.

6. Skrupky LP, McConnell K, Dallas J, et al. A comparison of ventilatorassociated pneumonia rates as identified according to the National Healthcare Safety Network and American College of Chest Physicians Criteria. Crit Care Med. 2012;40(1):281-284.

7. Saroj Golia, Sangeetha KT, Vasudha CL. Microbial profile of early and late onset ventilator associated pneumonia in the intensive care unit of a tertiary care hospital in bangalore, india. J Clin Diagn Res. 2013;7(11):2462-2466.

8. Pugin J, Auckenthaler R, Mili N, et al. Diagnosis of ventilatorassociated pneumonia by bacteriologic analysis of bronchoscopic and nonbronchoscopic "blind" bronchoalveolar lavage fluid. Am Rev Respir Dis. 1991;143(5 Pt 1):1121-1129.

9. Kalanuria AA, Ziai W, Mirski M. Ventilator-associated pneumonia in the ICU. Critical Care. 2014;18(2):208.

10. Wu CL, Yang DIe, Wang NY, et al. Quantitative culture of endotracheal aspirates in the diagnosis of ventilator-associated pneumonia in patients with treatment failure. Chest. 2002;122(2):662-668.

11. Cockerill F. Performance standards for antimicrobial susceptibility testing: twenty-second informational supplement. USA: Clinical and Laboratory Standards Institute; 2012.

12. Rasmussen BA, Bush K. Carbapenem-hydrolyzing beta-lactamases. Antimicrob Agents Chemother. 1997;41(2):223-232.
13. Jacoby GA. AmpC -Lactamases. Clin Microbiol Rev. 2009;22(1):161182.

14. Upadhyay S, Sen MR, Bhattacharjee A. Diagnostic utility of boronic acid inhibition with different cephalosporins against Escherichia coli producing AmpC -lactamases. J Med Microbiol. 2011;60(5):691-693.

15. Fagon JY, Chastre J, Domart Y, et al. Nosocomial pneumonia in patients receiving continuous mechanical ventilation. Prospective analysis of 52 episodes with use of a protected specimen brush and quantitative culture techniques. Am Rev Respir Dis. 1989;139(4):877-884.

16. Vallés J, Pobo A, García-Esquirol O, et al. Excess ICU mortality attributable to ventilator-associated pneumonia: the role of early vs late onset. Intensive Care Med. 2007;33(8):1363-1368.

17. Rello J, Ollendorf DA, Oster G, et al. Epidemiology and outcomes of ventilator-associated pneumonia in a large US database. Chest. 2002;122(6):2115-2121.

18. Dey A, Bairy I. Incidence of multi drug resistant organisms causing Ventilator associated pneumonia in a tertiary care hospital: A nine month prospective study. Ann Thorac Med. 2007;2(2):52-57.

19. Kumar MS, Lakshmi V, Rajagopalan R. Occurrence of extended spectrum $\beta$-lactamases among Enterobacteriaceae species isolated at a tertiary care institute. Indian J Med Microbial. 2006;24(3):208-211.

20. Jain S, Khety Z. Changing antimicrobial resistance pattern of isolates from an ICU over a 2year period. J Assoc Physicians India. 2012;60:2728.

21. Gaurav Dalela, Sweta Gupta, Dinesh Kumar Jain, et al. Antibiotic resistance pattern in uropathogens at a tertiary care hospital at jhalawar with special reference to esbl, ampc b-lactamase and mrsa production. Journal of Clinical and Diagnostic Research. 2012;6(4):645-651.

22. Rawat D, Nair D. Extended spectrum beta lactamases in Gram negative bacteria. J Glob Infect Dis. 2010;2(3):263-274.

23. Franklin C, Liolios, Peleg AY. Phenotypic detection of carbapenemsusceptible metallo-beta-lactamase-producing gram-negative bacilli in the clinical laboratory. J Clin Microbiol. 2006;44(9):3139-3144.

24. Oberoi L, Singh N, SharmaP, et al. ESBL, MBL and Ampc $\beta$ Lactamases Producing superbugs - Havoc in the Intensive Care Units of Punjab India. J Clin Diagn Res. 2013;7(1):7073. 